Proc. 8th European Symp. on Semiconductor Detectors

Schloss-Elmau, Germany

June $14-17,1998$

BNL-66891

\title{
Silicon Drift Detectors for the STAR/SVT experiment at RHIC
}

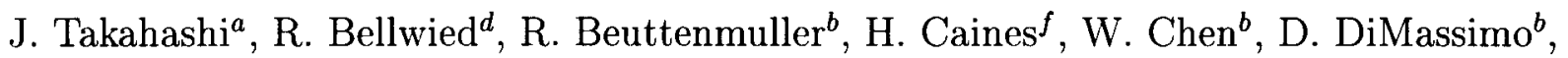

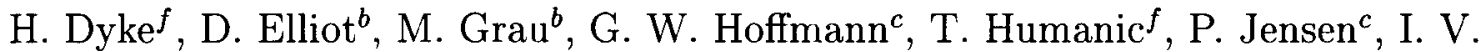

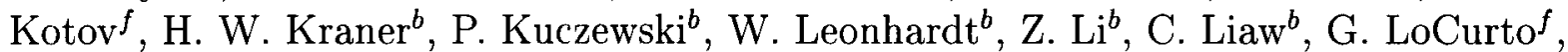
D. Lynn ${ }^{b}$, N. Mazeh ${ }^{d}$ P. Middelkamp ${ }^{b}$, R. Minor ${ }^{e}$, S. Nehmeh ${ }^{d}$ G. Ott ${ }^{b}$, S. U. Pandey ${ }^{d}$,

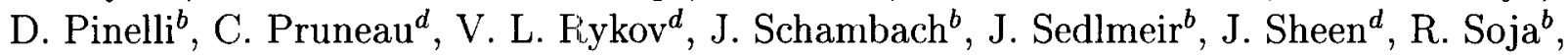
D. Stefani ${ }^{b}$, E. Sugarbaker ${ }^{f}$, and W. K. Wilson ${ }^{d}$

STAR-SVT Collaboration

a Universidade de São Paulo, São Paulo, Brazil

b Brookhaven National Laboratory, NY 11973, USA

c Universit; of Texas, Austin, TX 78712, USA

$d$ Wayne State University, MI 48201, USA

e Lawrence Berkeley Laboratory, CA 94720, USA

f Ohio State University, $\mathrm{OH} 43210$, USA

\begin{abstract}
Large area linear Silicon Drift Detectors (SDD) were developed to be used in the Silicon Vertex Tracker(SVT) of the STAR experiment at the BNL relativistic heavy ion collider (RHIC). The SDD is in its final design and has been submitted for large scale production. Test results show that the detector exibits excellent position resolution and low noise. A special characterization procedure was developed to test detector wafers in order to select good detectors for the SVT. Recently, 15 STAR/SVT SDD's were assembled as a tracking device in a BNL-AGS heavy ion experiment (E896). It is the first tracking application of these detectors and their corresponding front-end electronics in an experimental environment. Preliminary results indicating good detector performance are shown and discussed in this paper.
\end{abstract}

\section{Introduction}

Silicon Drift Detectors (SDD) [1, 2] provide unambiguous two dimensional position information for charged particle detection. The high spatial resolution with cost effective granularity make these devices ideal to be used as tracking detectors. Traditional position sensing Silicon detectors such as microstrip detectors or pixel detectors can provide relatively large area coverage and good position resolution. However, the number of readout channels increase proportionally with coverage and position resolution. The operating principle of the SDD is based upon creating a drift channel inside the depleted Silicon wafer. This combines the advantages of a semiconductor detector (e.g. large ionizations) with some of the best features of gas drift chambers (e.g. low number of readout channels). By measuring the drift time together with the distribution of signals along the readout anodes, position information can be obtained in both dimensions. 
A large area linear SDD was developed to be used in the Silicon Vertex Tracker (SVT), the inner tracking detector for the Solenoidal Tracker at RHIC (STAR) experiment [3]. The SVT consists of 216 Silicon drift detectors, each with an area of $6.3 \times 6.3 \mathrm{~cm}^{2}$, mounted on three concentric barrels around the interaction point. Wafers are mounted onto Beryllium ladder structures holding either 4,6 or 7 detectors depending on the barrel number. The ladders are arranged in a shape of polygon barrels with 8, 12 and 16 ladders each to encompass the colliding beams and their point of interaction at radii of $6.6,10.6$ and $14.5 \mathrm{~cm}$ respectively.

The final detector design minimized inactive area and improved the position resolution in order to maximize the tracking efficiency. In addition, two important system tests were recently performed with the STAR/SVT SDD's. A SVT integration test with four detectors assembled in a ladder configuration was successfully mounted and tested. Details of this test and results are described in a different paper [4]. Also, 15 SDD's were mounted as an array of tracking detectors to be used in a heavy ion experiment (BNL-AGS-E896). Some preliminary results from this experiment are described and discussed in this article.

\section{STAR/Silicon Drift Detector final design}

The STAR/SVT SDD's are produced using a 4 inch diameter, $280 \mu \mathrm{m}$ thick, neutron transmutation doped (NTD) Silicon wafer with an approximate resistivity of $3.0 \mathrm{k} \Omega \mathrm{cm}$. The detector part of the wafer has dimensions of $6.3 \times 6.3 \mathrm{~cm}^{2}$ and it is divided into two symmetrical drift directions. Each drift direction is composed of $220 p^{+}$implanted cathodes on both surfaces with a spatial pitch of $135 \mu \mathrm{m}$. In the charge collection region, $240 n^{+}$implanted anodes $250 \mu \mathrm{m}$ apart are surrounded by focusing cathodes. For drift velocity calibration, $2 \times 4$ MOS type charge injection lines were implemented between adjacent cathode strips at drift distances of $0.2 \mathrm{~cm}, 1.0 \mathrm{~cm}, 2.0 \mathrm{~cm}$ and at the maximum drift distance of $3.0 \mathrm{~cm}[5,6,7]$. Figure 1 shows a picture of a uncut Silicon wafer after detector processing. Further details of the detector design and fabrication can be found in $[8,9]$.

In this paper, we will discuss a few improvements between the final STAR-2.9 design and the previous STAR-2.7 prototype. In the new STAR-2.9 design, the guard area was slightly increased to better distribute the voltage drop on the lateral areas of the detector. The total active area of the detector was reduced from $96 \%$ to $94.5 \%$. However the increase of the guard area improved the voltage linearity and reduced the anode leakage current which resulted in a more reliable detector. In addition, the value of the implanted resistors was reduced from about $1 \mathrm{M} \Omega$ to about $0.5 \mathrm{M} \Omega$, to improve the "stiffness" of the resistor chain, reducing the voltage non-linearities caused by surface leakage currents. The 


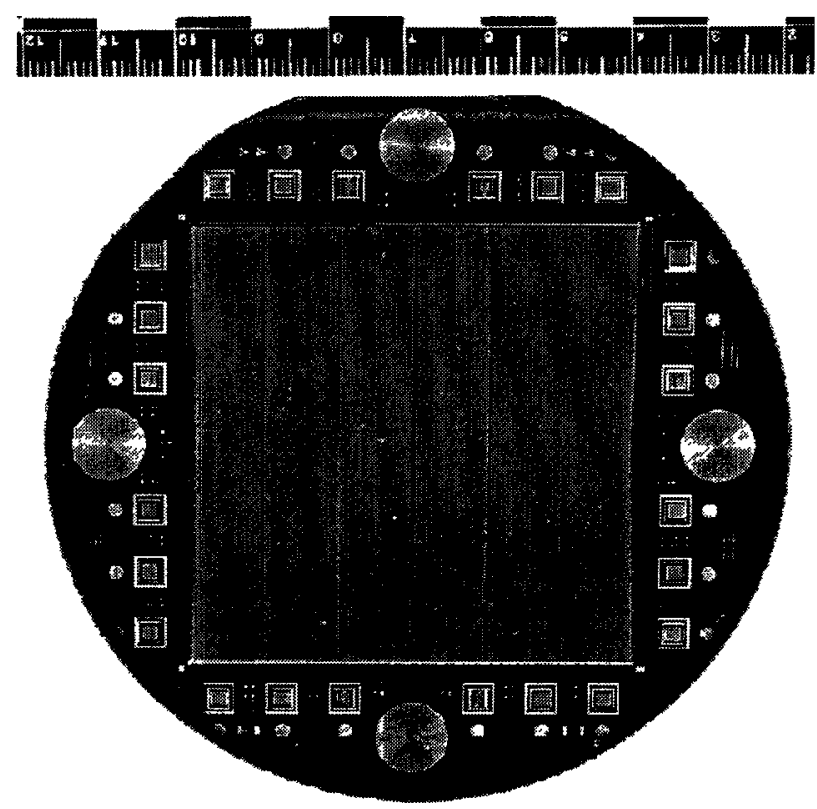

Figure 1: Picture of the bare Silicon wafer. The detector is processed on a 4 inch diameter NTD Silicon wafer, with active area of $6.3 \times 6.3 \mathrm{~cm}^{2}$.

design of the anode region was optimized to improve charge collection. Detectors from the STAR-2.7 batch have shown to be very sensitive to voltage variations of the focusing electrodes. In the STAR-2.9 detector the focusing region was modified, that improved the anode charge collection unifırmity [9]. On the STAR-2.9, the position of the charge injection lines were changed and the Aluminum overlay of the cathodes was widened.

Table 1 summarizes the quantilies and yields of previous STAR/SDD production, as well as for the future productions. Note the generally increasing production yield with subsequent design interations. In the development of the final detector design, the aim was not only to optimize the detector performance, but also to make the detector design sufficiently robust to increase the production yield. Delicate and thin structures that are sensitive to some processing steps such as etching were avoided in the design development. Oxide barriers between the anodes and the focusing electrode were increased to reduce possible voltage breakdowns. The STAR-2.7 detector was the first prototype produced in parallel by the Instrumentation Division at Brookhaven National Laboratory and SIN$\mathrm{TEF}$, Norway, a commercial vendor. 
Table 1: Summary of STAR/SVT-SDD prototypes production

\begin{tabular}{lllcc}
\hline Detector & Year & Produced by & Quantity produced & Production Yield \\
\hline STAR-1.0 & 1992 & BNL & 10 & $40 \%$ \\
STAR-2.0 & 1993 & BNL & 12 & $30 \%$ \\
STAR-2.5 & 1994 & BNL & 08 & $50 \%$ \\
STAR-2.7 & $1995-1996$ & BNL+SINTEF & 56 & $60 \%$ \\
STAR-2.8 & $1999-1997$ & BNL & 08 & $40 \%$ \\
STAR-2.9 & 1997 & BNL+SINTEF & 50 & $75 \%$ \\
STAR-2.9 & 1998 & BNL+SINTEF & 250 (expected) & $70 \%$ \\
STAR-2.9 & 1999 & BNL+SINTEF & 350 (expected) & $70 \%$ \\
\hline
\end{tabular}

With the detector design finalized, the wafer fabrication has now moved into production mode. The STAR/SVT requires approximately 250 wafers, including spares, for the conclusion of the SVT detector. Projecting a production yield of $70 \%$, it is expected to have all necessary detectors by the first half of the year 1999 .

\section{Production Mode Selection Criteria}

In large detector systems such as the SVT, which is composed of a considerable number of single detectors, it is important to verify that every detector to be mounted meets specifications. Dead areas on the detectors considerably reduce the tracking efficiency, and replacement of single wafers once the SVT is assembled is very difficult and undesirable. A testing procedure was developed to evaluate the quality of detector wafers and select them for the SVT assembly. This procedure has to be as complete as possible to provide reliable information on the functionality of the detectors, and has to be simple in order to allow the testing of a large quantity of wafers within a limited amount of time. The main challenge was to develop a non-destructive and low cost testing procedure, where bare wafers can be tested without wire bonding onto PC boards. To solve this problem, the wafers are tested on probe stations, where microscopic probes make electric connection to the detector.

The testing procedure can be separated into three main steps: visual inspection, test structure measurements and active region measurements. Visual inspection is performed during and after the processing of the detectors. During the fabrication, wafers are inspected between each major processing step. Standard defects such as electric bridges between electrodes or mask misalignment are searched for. In many cases, it is possible to redo certain processing steps and correct the defects. Once the detector is fabricated, a final inspection is performed. However due to the previous inspections, the rejection yield at this point is considerably low (below $5 \%$ ). 
The next step of the selection procedure consists of measurements performed on the test structures. On the outer edges of the wafer small structures such as implanted resistor chains, p-n diode junctions and MOS capacitors were implemented, as seen in figure 1. $\mathrm{I}-\mathrm{V}$ and $\mathrm{CV}$ measurements from these test structures provide valuable information such as oxide charge density, value of the implanted resistors and p-n junction reverse leakage current, that permit evaluation of the quality of the detector processing. Measurements are performed on a probe station instrumented with variable voltage sources, current meters and capacitance meters. In each probe station, all the electronic devices are controlled by a computer through GPIB drivers, which allows automated testing of the structures. Figures 2 and 3 show some typical results from resistor test structures I-V measurements and reversed bias diode I-V measurements, respectively. One of the curves in figure 2 (with solid circles) shows a voltage breakdown for bias values above $\pm 80 \mathrm{~V}$. For the SVT, the maximum bias on the detector is equivalent to a voltage difference of $7 \mathrm{~V}$ per resistor, which corresponds to $\pm 70 \mathrm{~V}$ in the scale of figure 2. However, to make a conservative selection, resistors are required to sustain voltages up to $\pm 100 \mathrm{~V}$. Diode $\mathrm{C}-\mathrm{V}$ measurements and MOS capacitor Flat Band voltage shift measurements are also performed on the test structure, but not shown here. For each of these measurements, a set of parameters and acceptable limits are defined as a detector selection criteria. The selection limits were defined based on experience with previous measurements and its correlation to the detector performance.

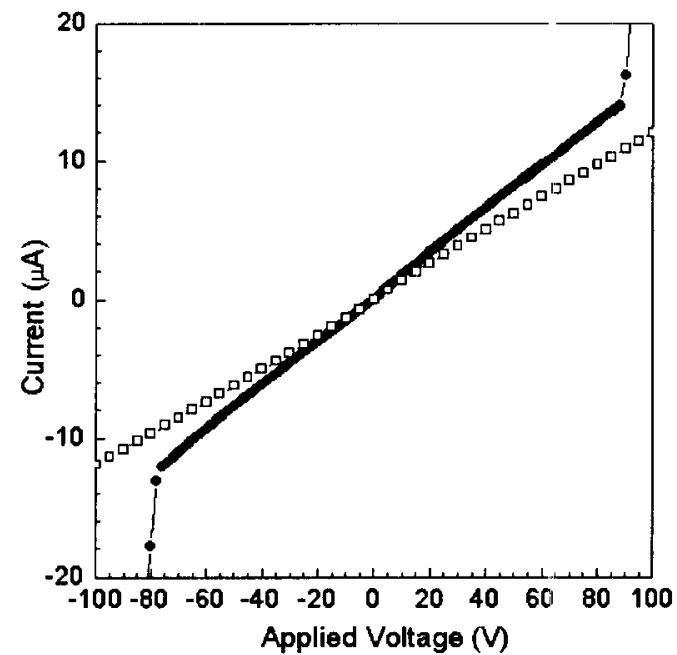

Figure 2: Typical I-V curve from resistor series test structures. Currents are measured from $-100 \mathrm{~V}$ to $+100 \mathrm{~V}$ for a series made of 10 implanted resistors.

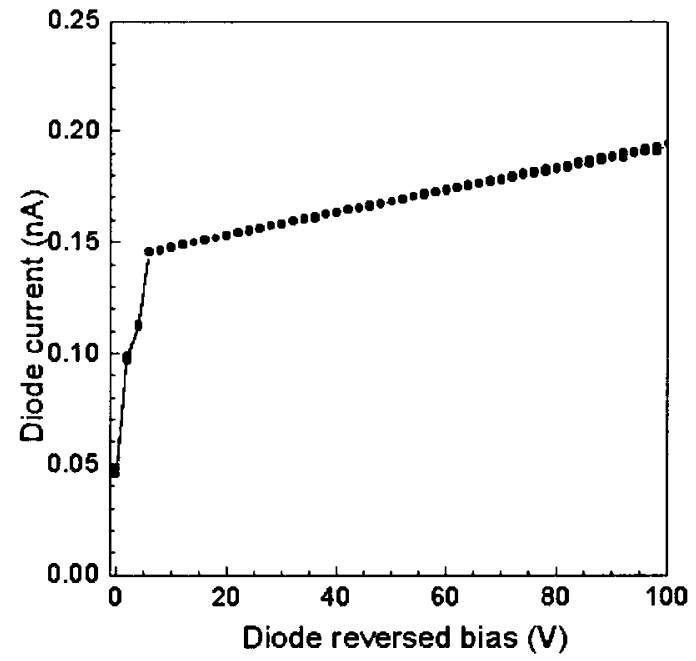

Figure 3: Typical I-V measurement from a p-n diode junction test structure. Maximum leakage current is required to be below $250 \mathrm{pA} / \mathrm{mm}^{2}$, diode area is $4 \mathrm{~mm}^{2}$. 
To illustrate the selection criteria, figures 4 and 5 show a summary of the test structure resistance measurement and reversed bias diode leakage current for a batch of 27 STAR-2.9 detectors. The straight horizontal line represent the limit of the selection criteria. From this detector batch, two wafers did not pass the resistor measurement criteria and three wafers showed diode leakage current above the accepted value. In this example, 4 out of 27 wafers did not pass our selection criteria, which corresponds to a yield of $85 \%$.

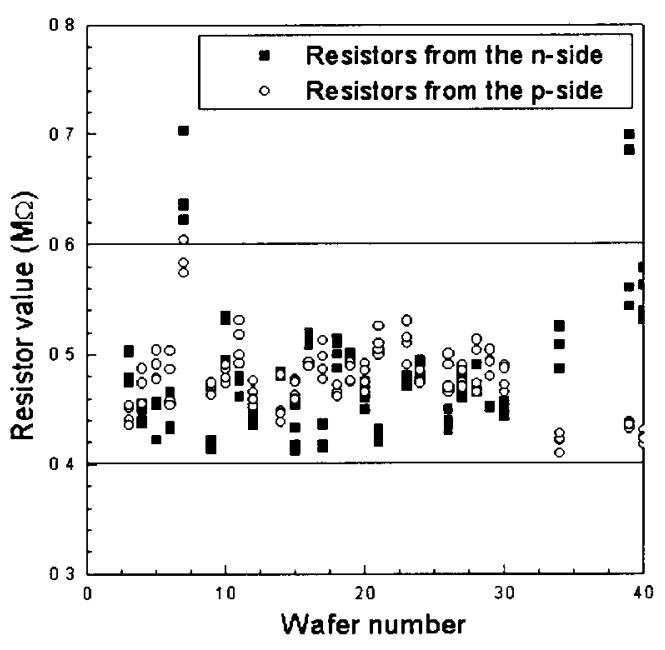

Figure 4: Summary of resistor test structure values measured from 27 detectors produced in the same batch. Horizontal lines indicate the criterion limits of $500 \pm 100 \mathrm{k} \Omega$.

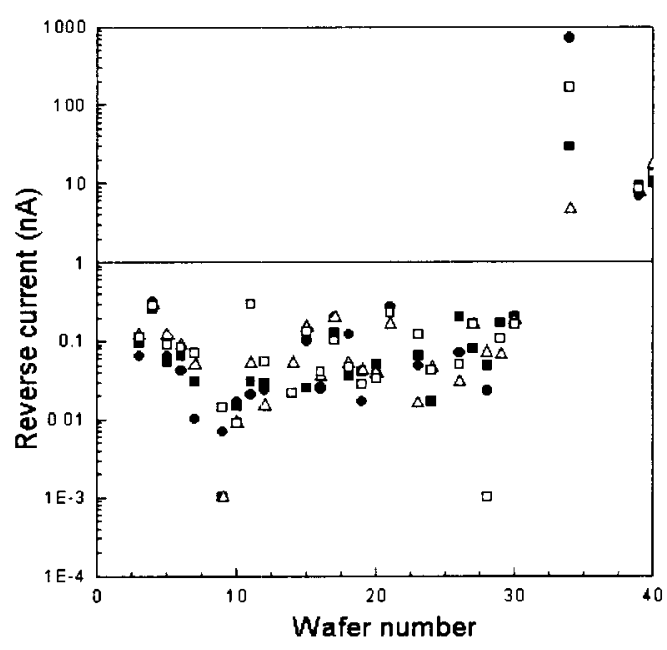

Figure 5: Summary of diode test structure current measurements at a reversed bias of $100 \mathrm{~V}$. The horizontal line indicates the maximum current limit of $1 \mathrm{nA}$.

In the next step, the active part of the wafer, i.e. the detector, is tested. Electrical measurements are performed on a special probe station. Probes on both sides of the detector make electrical contact with the surface of the detector, biasing the cathodes up to $1000 \mathrm{~V}$. Voltage linearity and guard anode currents are measured as a function of detector bias. On the anode side a 60 or 120 probe card is connected to the anodes to measure the leakage current. With this probe station, the active area of the detector can be tested without actually bonding the detector onto a PC board. Figure 6 shows typical results of the anode leakage current measured on the probe station. In this example, two wafers have anode leakage currents below the maximum acceptable value of $150 \mathrm{nA}$, and one detector (\# 23) shows a region with high leakage current. Figure 7 shows a summary of the average anode leakage currents from the batch of 27 detectors mentioned previously. Localized defects in the Silicon lattice can generate high leakage currents at the anodes. 


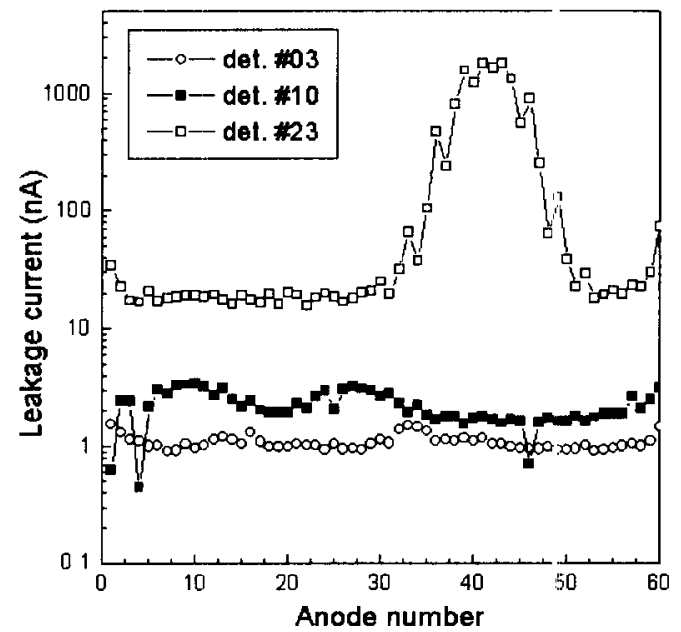

Figure 6: Typical results from I)C current measurement for 60 anodes from three different detectors measured on the probe station.

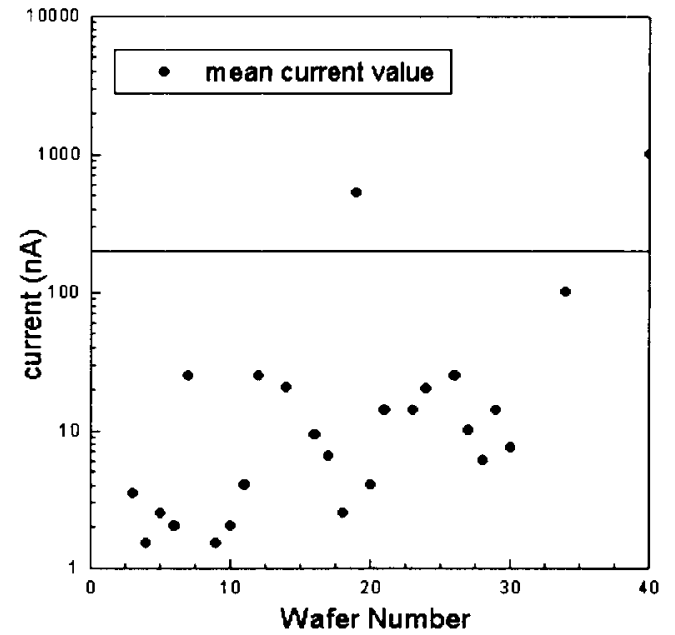

Figure 7: Summary of the average anode leakage current measured on the probe station with detectors biased at $1000 \mathrm{~V}$.

Based on the tests discussed in the previous sections, it is possible to determine the quality of the detector wafer in a non-destructive manner and assure that the selected wafers will perform as expected. From the 27 detectors tested, 7 detectors failed our criteria limits, resulting in a production yield of $74 \%$.

\section{Some results from STAR-2.9 detector}

During the detector series production, a few detectors per production batch are selected to be mounted on special testing P( ${ }^{\prime}$ boards for more detailed measurements. Laser injection through a microscope is used to ionize charges in a small spot (RMS width of $20 \mu \mathrm{m}$ ) on the detector, simulating a charged particle passing through the detector. An automated $\mathrm{X}-\mathrm{Y}$ stage moves the laser position on the detector which allows measurements such as drift linearity curves.

Figure 8 shows a typical result from a drift time, and drift non-linearity measurement taken on the laser test station. At the drift distance of approximately $3.0 \mathrm{~mm}$, there is a change in the drift velocity, caused by the intentional voltage difference between the 
$\mathrm{n}$-side and the $\mathrm{p}$-side of the focusing region of the detector (between cathode 20 and the anodes). Figure 9 shows a histogram of the drift non-linearity calculated with respect to a linear fit to the data above a drift distance of $3.0 \mathrm{~mm}$, out of the focusing region. In this example the non-linearity has a standard deviation of approximately $2.9 \mathrm{~ns}$, corresponding to a position non-linearity of approximately $20 \mu \mathrm{m}$. In a large sample of non-linearity measurements performed on various detectors, it was determined that most of the drift non-linearities are uniform across the anodes. Therefore a non-uniformity measurement from a few anodes is sufficient to "map" the detector.

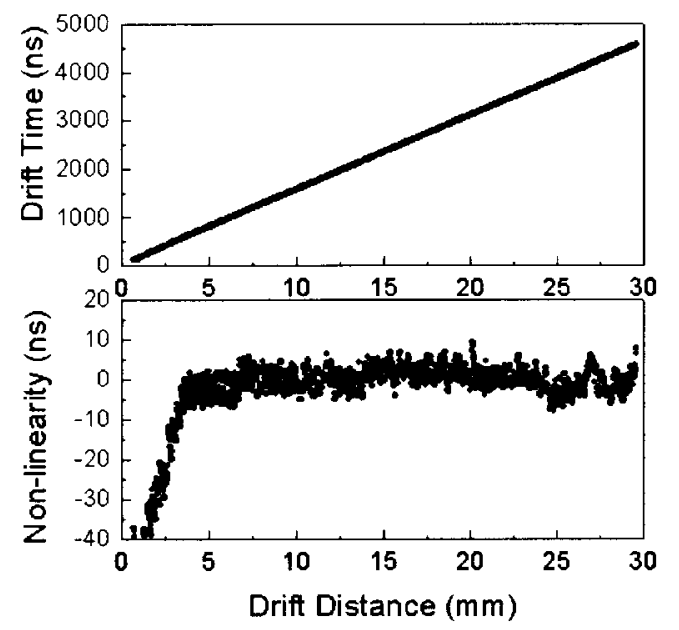

Figure 8: Drift time as a function of drift distance, measured on the laser test station.

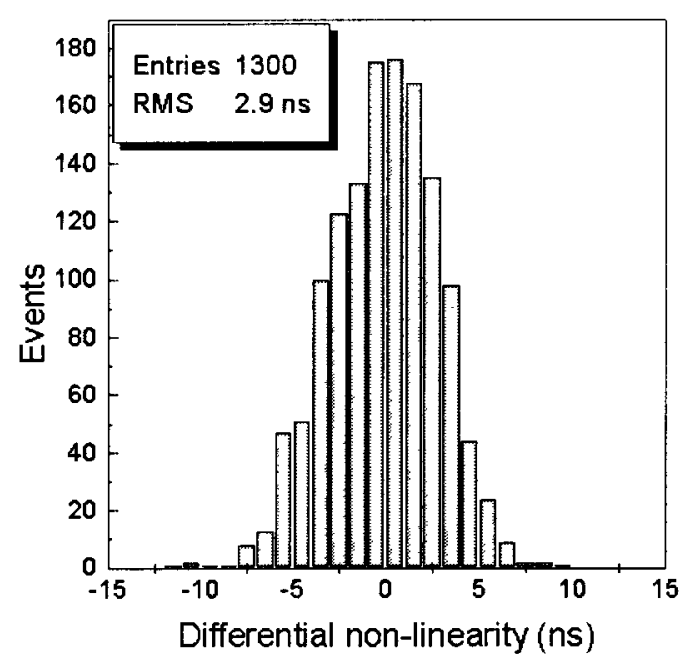

Figure 9: Distribution of the drift nonlinearities calculated from the deviation from a linear fit for drift distances above $3.0 \mathrm{~mm}$.

Intrinsic position accuracy in the drift direction is determined by taking a series of drift time measurements with the laser at fixed positions. Figure 10 shows the distribution of the position measurement for three different drift distances. The change in the accuracy with drift distance indicates that there is some systematic effect that affects the position accuracy, such as drift non-linearities and the decrease of the signal to noise ratio for longer drift distances. Position accuracy at the shortest drift distance corresponds to the intrinsic position accuracy of the detector and is $0.78 \mathrm{~ns}$. At full drift field of $6.2 \mu \mathrm{m} / \mathrm{ns}$ $(500 \mathrm{~V} / \mathrm{cm})$ it corresponds to a position accuracy of approximately $5 \mu \mathrm{m}$. The deviation of the drift time measurement at the maximum drift distance sets the minimum position accuracy achievable with the detector. 


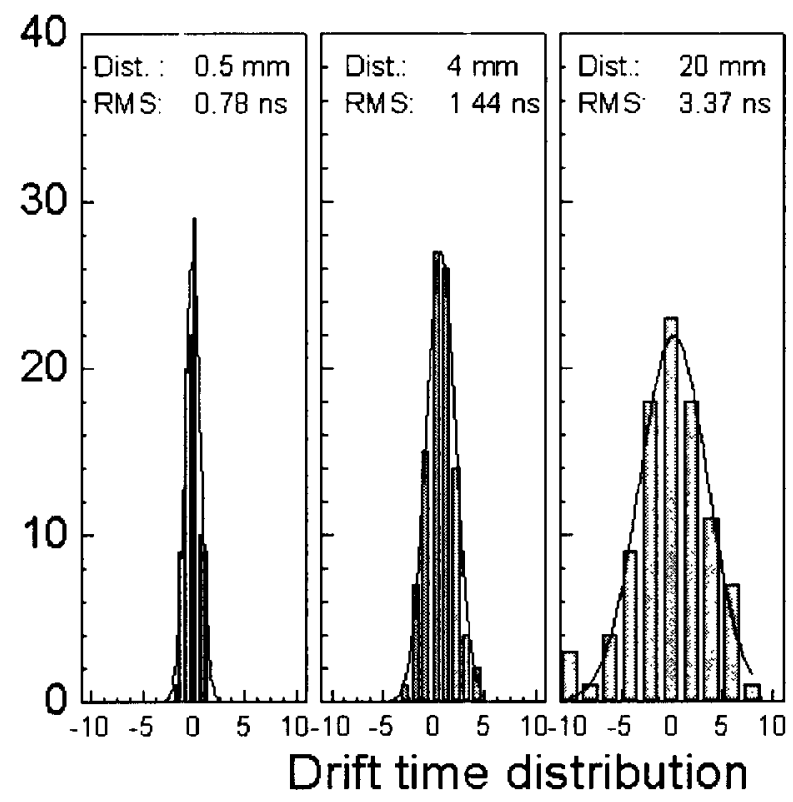

Figure 10: Scatter in the drift time measurement for three different drift distance. For each position, the measurement was sampled 100 times.

\section{Detector performance in the test beam}

The AGS Experiment E896 is a heavy-ion fixed target experiment measuring ${ }^{197} \mathrm{Au}+{ }^{197} \mathrm{Au}$ collisions at energy of $11.6 \mathrm{GeV} / \mathrm{c}$ per nucleon $[11,12]$. The experiment took place at the Alternating Gradient Synchrotron accelerator (AGS) of Brookhaven National Laboratory. The main physics motivation for this experiment is the search for the $\mathrm{H}_{0}$ dibaryon, an 6-quark state which is postulated to exist. The experiment also has high sensitivity for short lived particles which complements the previous AGS heavy-ion strangeness measurements by measuring particles such as $\Lambda, \bar{\Lambda}$, and $\Xi$ in $A u+A u$ collisions. The high sensitivity for short-lived particles is achieved by using an array of STAR/SVT Silicon Drift detectors for charged particle tracking and vertexing. The array is positioned near the interaction target, inside a superconducting magnet that generates a 6.2 Tesla field.

The drift detectors used in the experiment have been chosen using the same selection criteria used for STAR/SVT, described earlier in this paper. Detectors and their corresponding front-end electronics were mounted on custom printed circuit boards. Each detector was biased with an external voltage divider that was mounted on the PC-board and connected via wire bonds to every $10^{\text {th }}$ cathode on both sides of the detector. Front- 
end electronics assembled on a hybrid carrier were placed on both sides of the detector, aligned with the anodes and fixed using a heat conductive epoxy. Each anode on the detector was connected to an input channel of the pre-amplifier/shaper (PASA) though wire bonds.

The 15 PC-boards with detectors and front-end hybrids were held in position inside the magnet by a water cooled aluminum support structure. Figure 11 shows a picture of the Silicon drift detector array (SDDA) assembled with the 15 detectors used in the April 1998 data taking beam time.

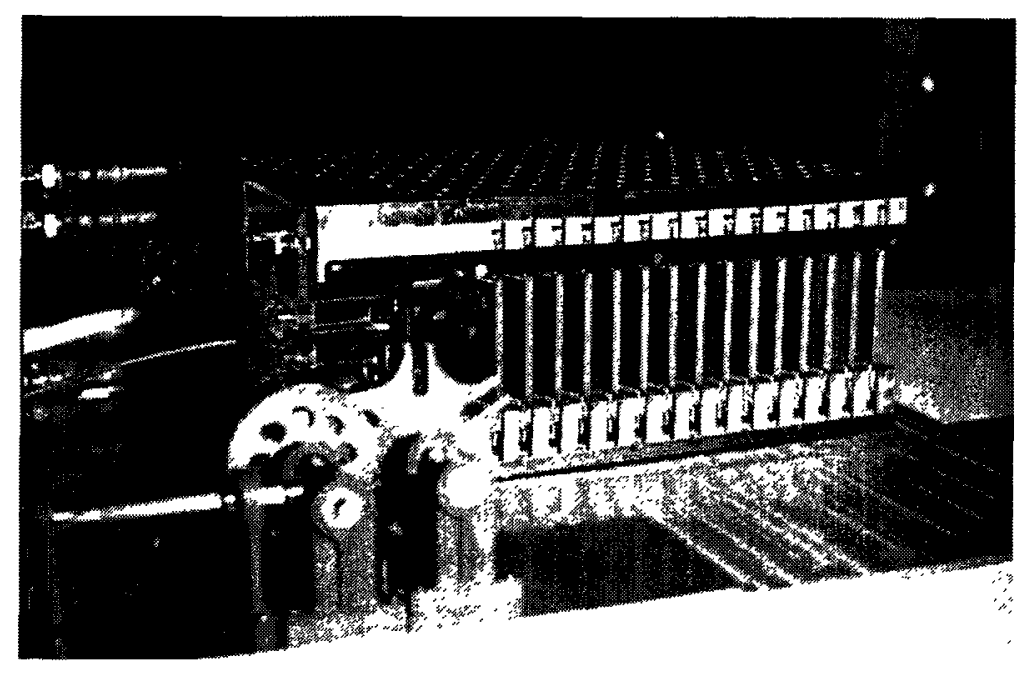

Figure 11: Picture of the Silicon Drift Detector Array assembled in the April 1998 beam run of AGS-BNL experiment 896.

Data were acquired using the same readout electronics and data-acquisition system to be used in the SVT. Approximately one million events were recorded over 25 days of data taking. During the data taking period, on-line monitoring software was used to view raw events, display hits on the detectors and calculate detector pedestals, noise levels and fluctuations. In addition, a slow control system monitored detector parameters such as guard anode current, and temperature.

The measured hit amplitude as a function of drift distance for a 100 events, is shown in figure 12. Each point of the scatter plot corresponds to a hit cluster amplitude. As expected the signal amplitude decreases with drift distance due to the diffusion of the electron cloud. Figure 13 shows the integrated charge of the measured cluster as a function of the drift distance. The points represented by solid squares include a correction based on an effect caused by the bipolar PASA response function. This result indicates that for most of the drift distance, the collected charge is independent of the drift distance, as expected. For short drift distance a slight increase in the integrated charge is observed. There are several possible causes for this increase. Inefficiency in measuring small clusters 
for short drift distances could caluse a shift of the most probable value of the integrated charge distribution for short drift distances. For short drift distances, there is little spread of the electron cloud therefore the signal is concentrated in a few pixels, instead of being distributed over many pixels. This problem is being solved by using a more sophisticated "hit finder" algorithm in the off-line analysis code.

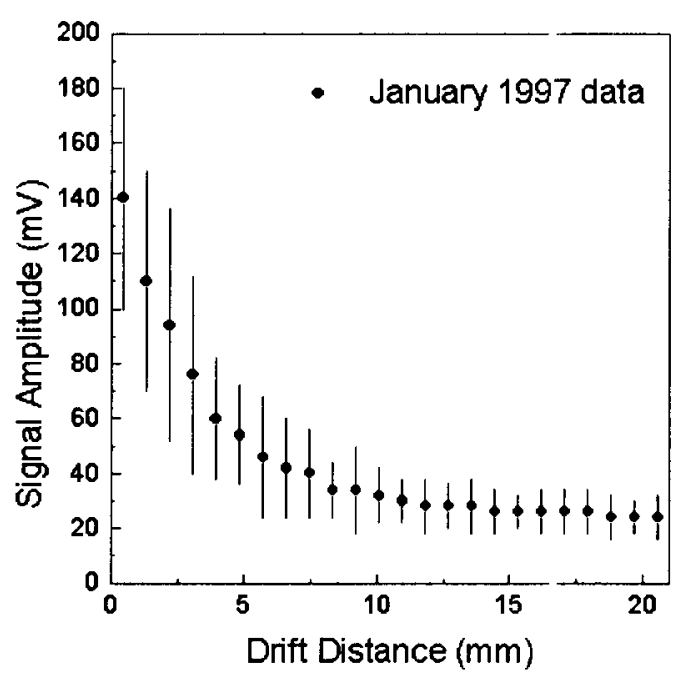

Figure 12: Measured cluster amplitude (highest pixel) as a function of drift distance.

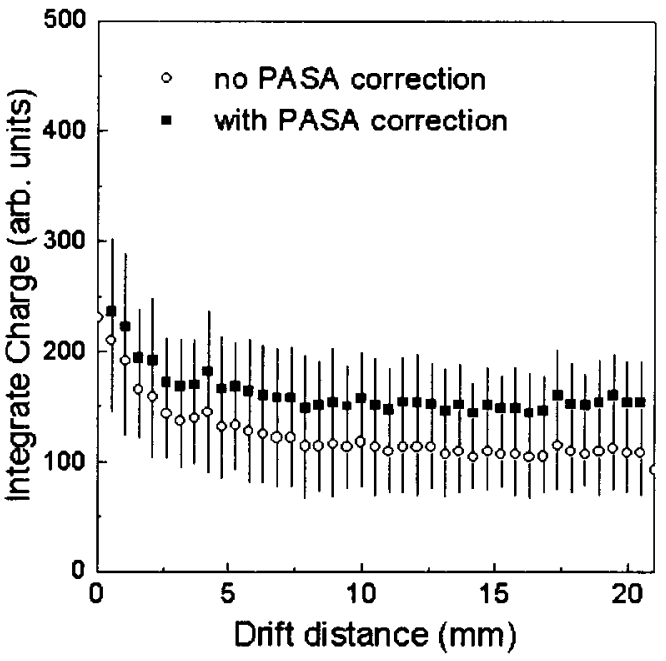

Figure 13: Most probable value of the surnmed ADC (total charge) in arbitrary units for different drift distances. The open circles correspond to the raw integrated ADC vales and the solid squares correspond to the summed ADC values corrected for the PASA response function effect.

The hit occupancy per event as a function of the detector coordinate in the transverse (anode) direction is shown in figure 14. The hit density increases towards the right side of the detector, where the bearı target was positioned. The solid circles represent the expected occupancy calculated from the simulation. The good agreement between the experimental data and the simulation indicates no hit detection inefficiency. Figure 15 shows the hit occupancy per event in the longitudinal (drift) direction and the comparison to the expected occupancy from the simulation. As expected, there is a higher density of hits in the center of the detector in the longitudinal direction where the beam was positioned.

Finally, figure 16 shows the number of hits measured in one of the detectors as a function of the event centrality (multiplicity) that was determined by a scintillation detector used in the experiment. The linear behavior of this dependence indicates that the number of hits increase proportionally with the centrality of reaction, with no evidence of any 


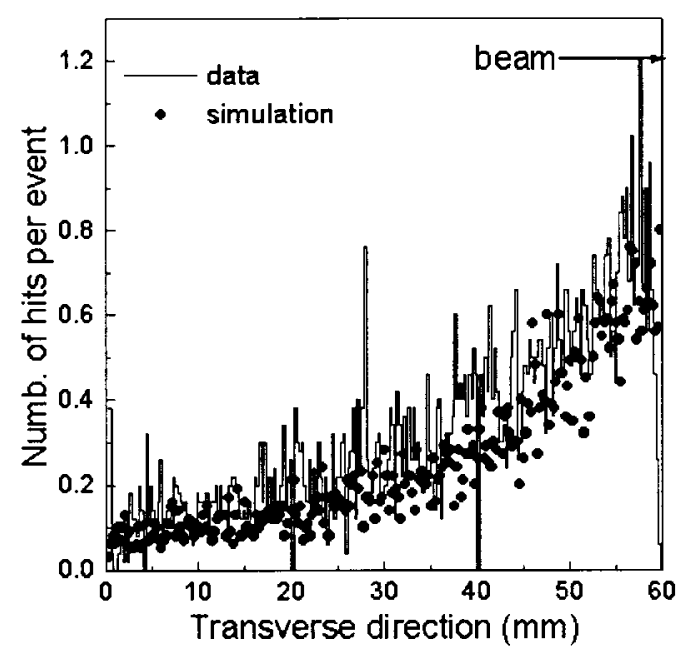

Figure 14: Detector hit occupancy in the transverse direction (histogram), compared to the expected occupancy from the simulation (solid circles).

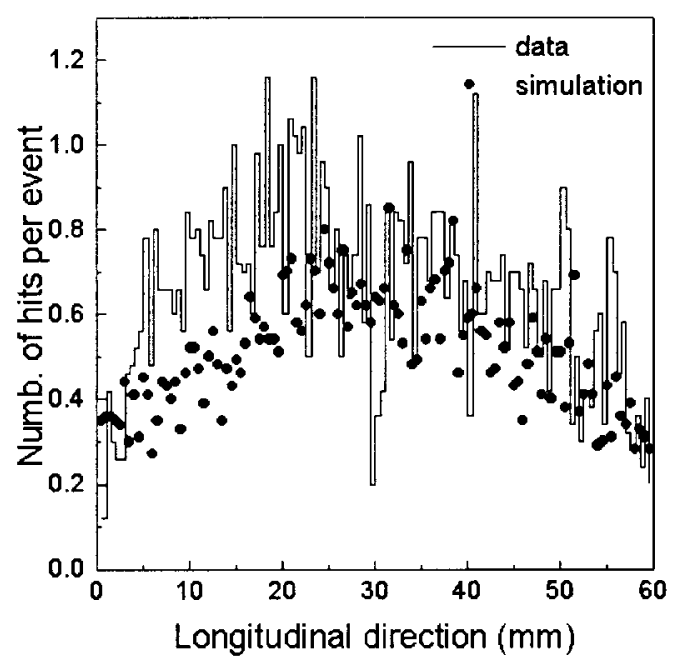

Figure 15: Detector hit occupancy in the longitudinal direction (histogram), compared to the expected occupancy from the simulation (solid circles).

saturation in high hit occupancy. For central collisions an average of 60 hits per event is measured on the detector.

The extremely high and relatively homogeneous magnetic field achievable with the superconducting magnet in E896 provided a unique opportunity to study effects of the magnetic field on the electron drift. Besides Hall effects, a magneto resistance effect is expected [10], due to changes in the lattice characteristics in a magnetic field. Data are now being analyzed.

\section{Conclusions}

The final detector design was concluded for the STAR/SVT SDD and submitted for commercial production. Tests on the laboratory bench, such as laser drift measurements and and anode leakage current measurements show that the detectors exibit excellent position resolution and low noise levels. Charge collection efficiency also shows good results.

Large scale production of these detectors has started in two different installations aiming to produce the 250 required wafers for the SVT. Due to the large quantity of wafers to be tested, and the high quality requirements of the SVT, a non-destructive testing procedure was developed to characterize and select the wafers produced to be mounted onto the SVT. Testing stations in two different institutions are capable of testing up to 3 


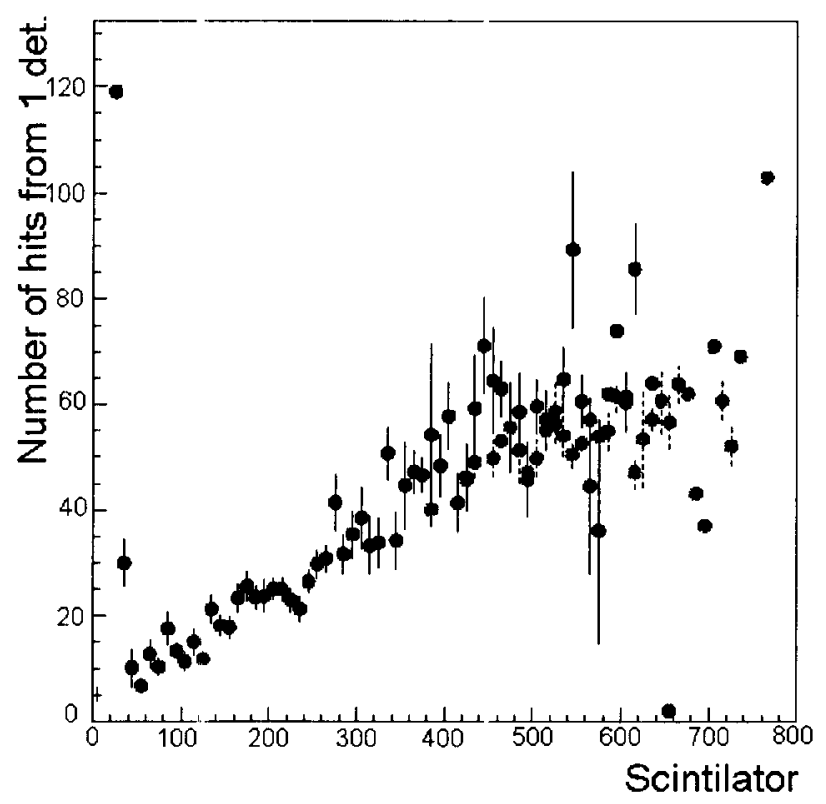

Figure 16: Relation between the number of hits measured in one Silicon Drift detector used in the AGS-E896 experiment and the reaction centrality, measured by a scintilator detector.

detector wafers per day.

In a recent heavy-ion experiment (AGS-E896) 15 SDD's were assembled in a high magnetic field environment and used as a tracking device. Preliminary analysis results indicate good detector performance, such $\downarrow$ as low noise levels and no evidence of charge recombination or hit loss. The total number of dead channels was around $1 \%, 80$ anodes out of 7200 anodes, which is below the SVT requirement of a maximum of $2 \%$ dead channels.

\section{Acknowledgements}

This work is supported in part by the US Department of Energy (under contract No. DEAC02-' 98CH10886 and DE-FQ02-92ER40713), the National Science Foundation (under grant PHY-9511850), and the Rubert A. Welch Foundation.

\section{References}

[1] E. Gatti and P. Rehak, Semiconductor Drift Chamber - An Application of a Novel Charge Transport Scheme, Nucl. Inst. and Meth. 225 (1984) 608. 
[2] P. Rehak et al., Semiconductor Drift Chambers for Position and Energy Measurements, Nucl. Inst. and Meth. A235 (1985) 224-234.

[3] J.W. Harris and the STAR Collaboration, The STAR Experiment at the Relativistic Heavy Ion Collider, Nucl. Phys. A566 (1994) 277c.

[4] D. Lynn et al., STAR/SVT Collaboration, A 240 Channel Thick Film Hybrid Circuit for Readout of Silicon Drift detectors, 8th European Symposium of Semiconductor Detectors, June 1998, Scholss Elmau, Germany.

[5] R. Bellwied et al., Electron Injection in Semiconductor Drift Detectors to be published in Nucl. Inst. and Meth. (1998).

[6] P. Rehak et al., Electron Injection in Semiconductor Drift Chambers, Conference Record IEEE NSS (Arlington, VA, Oct 22-27,1990) Vol 1, 41-45.

[7] E. Gatti et al., Electron injection in Semiconductor Drift Chambers, Nucl. Inst. and Meth. A295 (1990) 489-491.

[8] R. Bellwied et al., STAR/SVT Collaboration, Development of Large Linear Silicon Drift Detectors for the STAR Experiment at RHIC, Nucl. Inst. and Meth., A377 (1996) 387-392.

[9] R. Bellwied et al., Anode Region Design and Focusing Properties of STAR Silicon Drift Detectors, Nucl. Inst. and Meth., A400 (1997) 279-286.

[10] S. Pandey et al., STAR/SVT Collaboration, Transport Properties of Electrons in Silicon Measured with a Silicon Drift Detector in Large Magnetic Fields, Nucl. Inst. and Meth., A283 (1996) 537.

[11] H. Crawford, T.J. Hallman et al., Proposal 896 for BNL-AGS, Search for short-lived $\mathrm{H}$ dibarion, short-lived strange matter, and to investigate hyperon production in 11.6 $\mathrm{GeV} / \mathrm{c} / \mathrm{N} \mathrm{Au}+\mathrm{Au}$ collisions.

[12] W.J. Lope et al., The BNL-AGS Experiment $89612^{\text {th }}$ Winter Workshop on Nuclear Dynamics proceedings Snowbird, Utah, 1996, plenum press, New York. 